\title{
Knowledge about Essential Tremor: A Study of Essential Tremor Families
}

\author{
Ashley D. Cristal', Karen P. Chen ${ }^{1}$, Nora Cristina Hernandez', Pam Factor-Litvak², \\ Lorraine N. Clark ${ }^{3,4}$, Ruth Ottman ${ }^{2,5,6,7}$ and Elan D. Louis ${ }^{1,8,9 *}$
}

\begin{abstract}
'Division of Movement Disorders, Department of Neurology, Yale School of Medicine, Yale University, New Haven, CT, United States, ${ }^{2}$ Department of Epidemiology, Mailman School of Public Health, Columbia University, New York, NY, United States, ${ }^{3}$ Taub Institute for Research on Alzheimer's Disease and the Aging Brain, College of Physicians and Surgeons, Columbia University, New York, NY, United States, ${ }^{4}$ Department of Pathology and Cell Biology, Columbia University Medical Center, New York, NY, United States, ${ }^{5}$ G.H. Sergievsky Center, College of Physicians and Surgeons, Columbia University, New York, NY, United States, ${ }^{6}$ Department of Neurology, College of Physicians and Surgeons, Columbia University, New York, NY, United States, ' Department of Epidemiology, New York State Psychiatric Institute, New York, NY, United States, ${ }^{8}$ Department of Chronic Disease Epidemiology, Yale School of Public Health, Yale University, New Haven, CT, United States, ${ }^{9}$ Center for Neuroepidemiology and Clinical Neurological Research, Yale School of Medicine, Yale University, New Haven, CT, United States
\end{abstract}

OPEN ACCESS

Edited by:

Oscar Arias-Carrión,

Hospital General Dr. Manuel Gea

Gonzalez, Mexico

Reviewed by:

Laura Avanzino,

Università di Genova, Italy

Adán Miguel-Puga,

Universidad Nacional Autónoma de

México, Mexico

Miguel Coelho,

Universidade de Lisboa, Portugal

${ }^{*}$ Correspondence:

Elan D. Louis

elan.louis@yale.edu

Specialty section: This article was submitted to Movement Disorders, a section of the journal

Frontiers in Neurology

Received: 09 October 2017 Accepted: 12 January 2018

Published: 26 January 2018

Citation:

Cristal AD, Chen KP, Hernandez NC, Factor-Litvak $P$, Clark LN, Ottman $R$ and Louis ED (2018) Knowledge about Essential Tremor: A Study of Essential Tremor Families.

Front. Neurol. 9:27.

doi: 10.3389/fneur.2018.00027
Background: Essential tremor (ET) is among the most common neurological diseases and it often runs in families. How knowledgeable ET patients and their families are about their disease has been the subject of surprisingly little scholarship.

Methods: To fill this gap in knowledge, we administered a comprehensive 32-item survey (i.e., questions about etiology, pathophysiology, symptoms and signs, natural history, and treatments) to 427 participants, including 76 ET probands, 74 affected relatives (AFRs), 238 unaffected relatives, and 39 spouses of unaffected relatives, all of whom were participating in two ET family studies. We hypothesized that there would be gaps in knowledge about ET and furthermore, that probands and AFRs would be the most knowledgeable, followed by unaffected relatives and then spouses of unaffected relatives, who would be the least knowledgeable.

Results: Overall, ET patients lacked knowledge about their disease. Nearly one-third of probands answered "yes" or "do not know" to the question, "is ET the same or different from the type of tremor that many normal people can get when they become old and frail?" A similar proportion did not know whether children could get ET or they responded "no." Nearly one-fourth of affecteds (i.e., probands and AFRs) did not know whether or to what degree (e.g., very well, moderately well, not well) the symptoms of ET could be medically controlled, and $38.0 \%$ either reported that there was no brain surgery for ET or reported that they did not know. Nearly $17 \%$ of affecteds did not endorse genes as a cause for ET, which was surprising given the fact that this was a family study of ET. Probands and AFRs were the most knowledgeable, followed by unaffected relatives. Spouses of unaffected relatives were the least knowledgeable.

Conclusion: We targeted a large group of ET patients and their families, as this group is perhaps most likely to be informed about the disease. ET patients and their AFRs were more knowledgeable about the features of ET than their family members without ET. 
Overall, however, knowledge of ET was very limited and this lack of knowledge encompassed all aspects of the disease including its underlying causes, the nature of the symptoms and signs, its natural history and its treatment. Further ET awareness education and programs targeting both families of ET patients and the public would help alleviate this gap in knowledge.

Keywords: essential tremor, clinical, survey, epidemiology, genetics

\section{INTRODUCTION}

Essential tremor (ET) is among the most common movement disorders (1). New research on signs and symptoms, disease progression, risk factors, and treatment has expanded and changed our understanding of ET (2). For example, ET had previously been viewed as a monosymptomatic disorder, but a range of observed non-motor symptoms, such as cognitive impairment and sleep disturbances, has challenged this long-held view (3). For clinicians and patients, keeping up with new ET research findings can be challenging.

Despite being so common, ET is often misdiagnosed by physicians, with studies reporting misdiagnosis in $30-50 \%$ of cases (4, 5). A poll of 1,418 ET patients revealed that one-third believed physicians were not educated enough about ET (6). One-hundred and sixteen (9.5\%) believed physicians did not satisfactorily discuss and assess issues aside from tremor, and 146 (11.9\%) wanted better counseling and management (6). These data suggest that patients desire to learn more than their physicians are able to provide.

Increased knowledge of diseases can empower patients to have more control over their treatment; this can increase patient satisfaction, improve adherence to treatments, and yield better outcomes. In a systematic literature review of patient empowerment in cancer, the authors reported that knowledge is a key element of empowerment, and patients' understanding of their prognosis and treatment was essential for maintaining a sense of control and engaging in informed decision making (7). Others have reported that patients are more likely to act if they feel the activity has meaning (8). In the context of ET, patients have little control over their tremors and learning more about the disease could help empower them.

How knowledgeable ET patients and the public are regarding the disease has been the subject of only a handful of studies. Two studies focused solely on knowledge of the genetic basis of ET, concluding that most patients were poorly informed $(9,10)$. A third study examined public knowledge of ET using a more comprehensive questionnaire about the clinical features, causes of, and treatments for ET, but did not question patients themselves (11). No studies have administered a comprehensive questionnaire to ET patients themselves. Furthermore, none has focused exclusively on those with familial ET, which is a group that is more likely to be knowledgeable about the disease.

To fill this gap in knowledge, we administered a comprehensive survey to ET patients and their families. The survey incorporated questions about (1) etiology, genetic underpinnings, and familial risks, (2) pathophysiology, (3) symptoms and signs, (4) distinction from other conditions, (5) natural history and prognosis, (6) comorbidities, and (7) treatments. We surveyed both affected and unaffected family members as well as spouses of unaffected family members, hypothesizing that level of knowledge would follow a specific rank order depending on how distant a family member was from someone affected with ET.

\section{MATERIALS AND METHODS}

\section{Subjects and Setting}

The 427 participants were enrolled in two ET family studies at Yale University, a family study of the environmental epidemiology of ET (NINDS R01 NS094607, May 2016-present) and the Family Study of Essential Tremor (NINDS R01 NS073872, Phase 2, September 2015-present). The inclusion criteria and enrollment schemes for each study have been described in detail (12). Upon enrollment, participants signed informed consent approved by the Yale University Institutional Review Board. Each study involved a detailed clinical assessment of ET cases and their relatives (both affected and unaffected) using semi-structured questionnaires and a standardized videotaped neurological examination (12). The family study of the environmental epidemiology of ET included only first-degree relatives of a person with ET, whereas the Family Study of ET included both first-degree and seconddegree relatives. In the former, unaffected spouses of unaffected relatives were also enrolled. Based on the history and videotaped examination, a senior movement disorders neurologist (Elan D. Louis) assigned ET diagnoses using published diagnostic criteria [moderate or greater amplitude kinetic tremor on $\geq 3$ tests, or head tremor, in the absence of Parkinson's disease (PD) or dystonia] (12).

\section{ET Knowledge Questionnaire}

As part of their evaluation, all participants were also asked to complete a 32-item questionnaire assessing knowledge about ET. The survey was developed by one of the authors, Elan D. Louis, and included all of the disease-knowledge questions used in a questionnaire that was the centerpiece of a prior study (11) with the addition of 12 new questions relating to disease-knowledge. The questionnaire was piloted on $10 \mathrm{ET}$ patients to assess any ambiguities in wording, and then revised accordingly, thereby arriving at the final version used here. This questionnaire included single choice, multiple-selection, or fill-in-the-blank responses. The questionnaire began with demographic items, including occupation, since some occupations (i.e., health care-related professions) could lead participants to know more about ET. Next, the questionnaire focused on knowledge about (1) etiology, genetics, and familial risks, (2) pathophysiology, 
(3) symptoms and signs, (4) distinction from other conditions, (5) natural history and prognosis, (6) comorbidities, and (7) treatments.

\section{Statistical Analyses}

All analyses were performed in SPSS statistical software (version 24; SPSS Inc., Chicago, IL, USA). All tests were two-sided and significance was accepted at the $5 \%$ level.

Participants were grouped into four categories, with the purpose of determining whether they would differ with respect to knowledge of ET. These were ET probands $(n=76)$, affected first- or second-degree relatives [affected relatives (AFR), $n=74$ ], unaffected first- or second-degree relatives (UFR, $n=238$ ), and unaffected spouses of unaffected relatives $(n=39)$. The first two groups were assessed separately because of the possibility that probands (i.e., individuals who had been motivated enough to self-refer for participation in the study) might be more knowledgeable about ET.

Age and education were not normally distributed (Kolmogorov-Smirnov test $p<0.001$ for both). We compared age and education across groups using Kruskall-Wallis tests, and used chi-square tests (or Fisher's exact test) for additional comparisons of categorical data. To test for trends across the four subject type groups (probands $>$ AFRs $>$ UFRs $>$ spouses), we used logistic regression models, with the response as a binary dependent variable and the four ordinally arranged subject type groups (probands $=1, \mathrm{AFRs}=2, \mathrm{UFRs}=3$, and spouses $=4$ ) as the independent variable. Although this yielded odds ratios, 95\% confidence intervals, and $p$ values, we only report the latter.

\section{RESULTS}

\section{Demographic Characteristics}

There were 427 participants (76 probands, 74 AFRs, 238 UFRs, and 39 spouses of UFRs) (Table 1). Probands were on average approximately 6 years older than other participants. On average, participants completed 16 years of education. Sixty (14.1\%) were in a health-related occupation. Among probands, the mean age of tremor onset was $29.2 \pm 19.8$ years (range $=4-77$ years) and the mean tremor duration was $37.5 \pm 17.7$ years (range $=1-77$ years).

\section{Etiology, Genetics, and Familial Risks}

Three-quarters $[325 / 427(76.1 \%)]$ of the participants endorsed "genes" as a cause for ET. At the same time, 25/150 (16.7\%) of affecteds (i.e., probands and AFRs) did not do so, a finding that is somewhat surprising given the fact that this was a family study of ET (Table 2). More than one-third (15/39, 38.5\%) of spouses did not endorse "genes" as a cause of ET either. Indeed, there was a clear trend across the four subject type groups ("genes" as a cause of ET in $88.2 \%$ of probands, $78.4 \%$ of AFRs, $73.9 \%$ of UFR, and $61.5 \%$ of spouses, Table 2 ) (test for trend, $p=0.001$ ). Only $97 / 427$ (22.7\%) participants responded that genes had been identified for ET, which included only 23/76 (30.3\%) probands (Table 2). Virtually none of the participants knew that specific genes have been reported as associated with ET in scientific studies (e.g., LINGO1, FUS, ETM) (Table 2).

Fifty-five percent of participants (237/427) and 31/76 (40.8\%) probands did not know the level to which first-degree relatives of affected persons were at increased risk for ET. Indeed, a majority of relatives, including both AFRs and UFRs (180/312 = 57.7\%), did not know the level (Table 2). There was a clear trend across the four subject type groups ("did not know" in $40.8 \%$ of probands, $47.3 \%$ of AFRs, $60.9 \%$ of UFRs, and $66.7 \%$ of spouses, Table 2 ) (test for trend, $p<0.001$ ).

\section{Pathophysiology}

Nearly one-half of probands $(37 / 76,48.7 \%)$ considered ET to be a neurodegenerative disease (Table 2). Although the thalamus and cerebellum were the two regions most endorsed as the brain region responsible for ET (from a list of seven brain regions), even among probands, only one-fourth or fewer endorsed each of these brain regions (Table 2).

\section{Symptoms and Signs}

Nearly all participants [415/427 (97.2\%)] identified the upper limbs as a part of the body that can shake in ET patients while $81 \%$ (346/427) identified cranial structures. For cranial structures, there was a clear trend across the four subject type groups (yes to "cranial tremor" in $90.8 \%$ of probands, $87.8 \%$ of AFRs, $79.4 \%$ of UFRs, and $59.0 \%$ of spouses, Table 2) (test for trend, $p<0.001$ ). Most participants $(266 / 427,62.3 \%)$ and most probands $(48 / 76$,

TABLE 1 | Demographic characteristics of 427 participants.

\begin{tabular}{|c|c|c|c|c|c|c|}
\hline & Proband & Affected relative & Unaffected relative & Spouse of unaffected relative & All participants & Significance \\
\hline$N$ & 76 & 74 & 238 & 39 & 427 & \\
\hline Age in years & $\begin{array}{r}66.2 \pm 12.0 \\
\text { Median } 66.5\end{array}$ & $\begin{array}{r}61.2 \pm 16.9 \\
\text { Median } 65.5\end{array}$ & $\begin{array}{l}58.0 \pm 13.5 \\
\text { Median } 58.0\end{array}$ & $\begin{array}{l}60.1 \pm 11.0 \\
\text { Median } 61.0\end{array}$ & $\begin{array}{l}60.2 \pm 14.0 \\
\text { Median } 61.0\end{array}$ & $p<0.001^{\mathrm{a}}$ \\
\hline Female gender & $50(65.8)$ & $42(56.8)$ & $159(66.8)$ & $14(35.9)$ & $265(62.1)$ & $p=0.002^{b}$ \\
\hline Education in years & $\begin{array}{c}15.8 \pm 2.9 \\
\text { Median } 16.0\end{array}$ & $\begin{array}{c}15.6 \pm 2.9 \\
\text { Median } 16.0\end{array}$ & $\begin{array}{c}16.4 \pm 2.9 \\
\text { Median } 16.0\end{array}$ & $\begin{array}{c}15.6 \pm 5.5 \\
\text { Median } 16.0\end{array}$ & $\begin{array}{c}16.0 \pm 3.2 \\
\text { Median } 16.0\end{array}$ & $p=0.026^{a}$ \\
\hline Health-related occupation ${ }^{c}$ & $9(11.8)$ & $6(8.1)$ & $41(17.2)$ & $4(10.3)$ & $60(14.1)$ & $p=0.177^{b}$ \\
\hline
\end{tabular}

Number of participants (percentage) or mean $\pm S D$. For age and education, we also report the median.

${ }^{a}$ Kruskal-Wallis test.

${ }^{b}$ Chi-square test.

cPhysician $(n=1)$, nurse $(n=16)$, home health aide $(n=2)$, student $(n=2)$, medical research $(n=6)$, and other healthcare occupation $(n=33)$. 
TABLE 2 | Responses of 427 participants to 28 questions about the essential tremor (ET).

\begin{tabular}{|c|c|c|c|c|c|c|}
\hline & Proband & $\begin{array}{l}\text { Affected } \\
\text { relative }\end{array}$ & $\begin{array}{l}\text { Unaffected } \\
\text { relative }\end{array}$ & $\begin{array}{c}\text { Spouse of } \\
\text { unaffected relative }\end{array}$ & All participants & Significance \\
\hline$N$ & 76 & 74 & 238 & 39 & 427 & \\
\hline \multicolumn{7}{|c|}{ What do you think causes ET? (Indicate one or more) } \\
\hline Genes & $67(88.2)$ & $58(78.4)$ & $176(73.9)$ & $24(61.5)$ & $325(76.1)$ & $p=0.010^{\mathrm{a}}$ \\
\hline Trauma & $9(11.8)$ & $4(5.4)$ & $16(6.7)$ & $0(0.0)$ & $29(6.8)$ & $p=0.105^{\mathrm{a}}$ \\
\hline Brain disease & $10(13.2)$ & $7(9.5)$ & $27(11.3)$ & $2(5.1)$ & $46(10.8)$ & $p=0.394^{\mathrm{a}}$ \\
\hline Abnormal dietary habits & $1(1.3)$ & $4(5.4)$ & $26(10.9)$ & $2(5.1)$ & $33(7.7)$ & $p=0.033^{a}$ \\
\hline Alcohol abuse & $6(7.9)$ & $8(10.8)$ & $17(7.1)$ & $1(2.6)$ & $32(7.5)$ & $p=0.457^{\mathrm{a}}$ \\
\hline Tobacco abuse & $0(0.0)$ & $1(1.4)$ & $7(2.9)$ & $1(2.6)$ & $9(2.1)$ & $p=0.443^{\mathrm{a}}$ \\
\hline Environmental toxins & $8(10.5)$ & $8(10.8)$ & $32(13.4)$ & $2(5.1)$ & $50(11.7)$ & $p=0.477^{\mathrm{a}}$ \\
\hline Medication/drugs & $6(7.9)$ & $6(8.1)$ & $19(8.0)$ & $0(0.0)$ & $31(7.3)$ & $p=0.339^{a}$ \\
\hline Systemic metabolic diseases & $3(3.9)$ & $2(2.7)$ & $15(6.3)$ & $0(0.0)$ & $20(4.7)$ & $p=0.255^{\mathrm{a}}$ \\
\hline Combination of more than one of the above & $17(22.4)$ & $14(18.9)$ & $68(28.6)$ & $12(30.8)$ & $111(26.0)$ & $p=0.292^{\mathrm{a}}$ \\
\hline Unknown cause & $23(30.3)$ & $24(32.4)$ & $73(30.7)$ & $9(23.1)$ & $129(30.2)$ & $p=0.768^{\mathrm{a}}$ \\
\hline Have scientists discovered genes for ET? & & & & & & $p<0.001^{a}$ \\
\hline Yes & $23(30.3)$ & $16(21.6)$ & $53(22.3)$ & $5(12.8)$ & $97(22.7)$ & \\
\hline No & $28(36.8)$ & $12(16.2)$ & $28(11.8)$ & $7(17.9)$ & $75(17.6)$ & \\
\hline Don't know & $25(32.9)$ & $46(62.2)$ & $157(66.0)$ & $27(69.2)$ & $255(59.7)$ & \\
\hline \multicolumn{7}{|c|}{ Have any of the following genes been linked with ET in scientific studies? } \\
\hline LRRK2 & $0(0.0)$ & $1(1.4)$ & $0(0.0)$ & $0(0.0)$ & $1(0.2)$ & $p=0.096^{\mathrm{a}}$ \\
\hline BCDN2 & $0(0.0)$ & $0(0.0)$ & $0(0.0)$ & $0(0.0)$ & $0(0.0)$ & $p=0.086^{\mathrm{a}}$ \\
\hline LINGO1 & $1(1.3)$ & $0(0.0)$ & $10(4.2)$ & $0(0.0)$ & $11(2.6)$ & $p=0.031^{a}$ \\
\hline ACTY7 & $0(0.0)$ & $0(0.0)$ & $0(0.0)$ & $0(0.0)$ & $0(0.0)$ & $p=0.086^{a}$ \\
\hline FUS & $0(0.0)$ & $0(0.0)$ & $1(0.4)$ & $0(0.0)$ & $1(0.2)$ & $p=0.232^{\mathrm{a}}$ \\
\hline MAPT & $0(0.0)$ & $0(0.0)$ & $1(0.4)$ & $0(0.0)$ & $1(0.2)$ & $p=0.232^{\mathrm{a}}$ \\
\hline ETM & $0(0.0)$ & $0(0.0)$ & $2(0.8)$ & $0(0.0)$ & $2(0.5)$ & $p=0.183^{\mathrm{a}}$ \\
\hline DRD3 & $0(0.0)$ & $1(1.4)$ & $2(0.8)$ & $0(0.0)$ & $3(0.7)$ & $p=0.248^{a}$ \\
\hline HS1-BP3 & $0(0.0)$ & $0(0.0)$ & $2(0.8)$ & $0(0.0)$ & $2(0.5)$ & $p=0.183^{\mathrm{a}}$ \\
\hline \multicolumn{6}{|c|}{ If someone has a parent or sibling with ET, their risk of developing ET is how much higher than someone with ET? } & $p=0.151^{\mathrm{a}}$ \\
\hline 0x (they don't have a higher risk) & $9(11.8)$ & $9(12.2)$ & $16(6.7)$ & $4(10.3)$ & $38(8.9)$ & \\
\hline $1.5 \times$ & $2(2.6)$ & $1(1.4)$ & $6(2.5)$ & $3(7.7)$ & $12(2.8)$ & \\
\hline $2 \times$ & $10(13.2)$ & $8(10.8)$ & $19(8.0)$ & $1(2.6)$ & $38(8.9)$ & \\
\hline $3 \times$ & $3(3.9)$ & $2(2.7)$ & $6(2.5)$ & $1(2.6)$ & $12(2.8)$ & \\
\hline $4 x$ & $2(2.6)$ & $0(0.0)$ & $4(1.7)$ & $0(0.0)$ & $6(1.4)$ & \\
\hline $5 \times$ & $4(5.3)$ & $8(10.8)$ & $15(6.3)$ & $2(5.1)$ & $29(6.8)$ & \\
\hline $6 x$ & $0(0.0)$ & $1(1.4)$ & $0(0.0)$ & $0(0.0)$ & $1(0.2)$ & \\
\hline $8 x$ & $0(0.0)$ & $0(0.0)$ & $2(0.8)$ & $0(0.0)$ & $2(0.5)$ & \\
\hline $10 x$ & $2(2.6)$ & $4(5.4)$ & $8(3.4)$ & $1(2.6)$ & $15(3.5)$ & \\
\hline More than $10 x$ & $13(17.1)$ & $6(8.1)$ & $17(7.1)$ & $1(2.6)$ & $37(8.7)$ & \\
\hline Don't know & $31(40.8)$ & 35 (47.3) & $145(60.9)$ & $26(66.7)$ & 237 (55.5) & \\
\hline Is ET a neurodegenerative disease? & & & & & & $p=0.026^{a}$ \\
\hline Yes & $37(48.7)$ & 27 (36.5) & $92(38.7)$ & $12(30.8)$ & 168 (39.3) & \\
\hline No & $16(21.1)$ & 9 (12.2) & $24(10.1)$ & $5(12.8)$ & $54(12.6)$ & \\
\hline Don't know & $23(30.3)$ & $38(51.4)$ & $122(51.3)$ & $22(56.4)$ & 205 (48.0) & \\
\hline \multicolumn{7}{|c|}{ For each of the brain structures, indicate whether scientists believe there is a problem in patients with ET } \\
\hline Thalamus & $19(25.0)$ & $6(8.1)$ & $35(14.7)$ & $2(5.1)$ & $62(14.5)$ & $p=0.044^{\mathrm{a}}$ \\
\hline Sensory cortex & $5(6.6)$ & $4(5.4)$ & $17(7.1)$ & $4(10.3)$ & $30(7.0)$ & $p=0.172^{\mathrm{a}}$ \\
\hline Cerebellum & $16(21.1)$ & $7(9.5)$ & $25(10.5)$ & $1(2.6)$ & 49 (11.5) & $p=0.003^{a}$ \\
\hline Inferior olivary nucleus & $2(2.6)$ & $1(1.4)$ & $4(1.7)$ & $0(0.0)$ & $7(1.6)$ & $p=0.286^{\mathrm{a}}$ \\
\hline Spinal cord & $2(2.6)$ & $2(2.7)$ & $8(3.4)$ & $0(0.0)$ & $12(2.8)$ & $p=0.196^{\mathrm{a}}$ \\
\hline Red nucleus & $1(1.3)$ & $1(1.4)$ & $2(0.8)$ & $0(0.0)$ & $4(0.9)$ & $p=0.206^{\mathrm{a}}$ \\
\hline Supplementary motor area & $7(9.2)$ & $8(10.8)$ & $23(9.7)$ & $8(20.5)$ & $46(10.8)$ & $p=0.072^{\mathrm{a}}$ \\
\hline \multicolumn{7}{|l|}{ What are the main symptoms of ET? } \\
\hline Tremors (unspecified) & $36(47.4)$ & $36(48.6)$ & $84(35.3)$ & $11(28.2)$ & $167(39.1)$ & $p=0.038^{\mathrm{a}}$ \\
\hline Shaky arms/hands & $37(48.7)$ & $33(44.6)$ & $153(64.3)$ & $23(59.0)$ & $246(57.6)$ & $p=0.007^{a}$ \\
\hline Shaky head & $21(27.6)$ & $15(20.3)$ & $77(32.4)$ & $9(23.1)$ & $122(28.6)$ & $p=0.189^{a}$ \\
\hline Shaky legs/feet & $5(6.6)$ & $4(5.4)$ & $24(10.1)$ & $1(2.6)$ & $34(8.0)$ & $p=0.276^{a}$ \\
\hline Shaky voice & $18(23.7)$ & $11(14.9)$ & $54(22.7)$ & $6(15.4)$ & $89(20.8)$ & $p=0.366^{\mathrm{a}}$ \\
\hline Other & $17(22.4)$ & $12(16.2)$ & $48(20.2)$ & $6(15.4)$ & $83(19.4)$ & $p=0.706^{\mathrm{a}}$ \\
\hline Do not know & $2(2.6)$ & $3(4.1)$ & $10(4.2)$ & $3(7.7)$ & $18(4.2)$ & $p=0.649^{a}$ \\
\hline \multicolumn{7}{|c|}{ What parts of the body can shake when someone has ET? } \\
\hline Arms (with or without other) & $73(96.1)$ & $71(95.9)$ & $233(97.9)$ & $38(97.4)$ & $415(97.2)$ & $p=0.598^{\mathrm{a}}$ \\
\hline Legs (with or without other) & $45(59.2)$ & $35(47.3)$ & $77(32.4)$ & $7(17.9)$ & $164(38.4)$ & $p<0.001^{a}$ \\
\hline Cranial structures (with or without other) & $69(90.8)$ & $65(87.8)$ & $189(79.4)$ & $23(59.0)$ & $346(81.0)$ & $p<0.001^{a}$ \\
\hline
\end{tabular}


TABLE 2 | Continued

\begin{tabular}{|c|c|c|c|c|c|c|}
\hline & Proband & $\begin{array}{l}\text { Affected } \\
\text { relative }\end{array}$ & $\begin{array}{l}\text { Unaffected } \\
\text { relative }\end{array}$ & $\begin{array}{c}\text { Spouse of } \\
\text { unaffected relative }\end{array}$ & All participants & Significance \\
\hline Total body & 17 (22.4) & 12 (16.2) & 30 (12.6) & $0(0.0)$ & 59 (13.8) & $p=0.030^{\mathrm{a}}$ \\
\hline \multicolumn{6}{|c|}{ Do patients with ET have problems with balance and walking that are greater than people without ET? } & $p=0.665^{\mathrm{a}}$ \\
\hline Yes & 48 (63.2) & $46(62.2)$ & 149 (62.6) & $23(59.0)$ & 266 (62.3) & \\
\hline No & $13(17.1)$ & $7(9.5)$ & 34 (14.3) & 4 (10.3) & 58 (13.6) & \\
\hline Don't know & 15 (19.7) & $21(28.4)$ & $55(23.1)$ & $12(30.8)$ & $103(24.1)$ & \\
\hline \multicolumn{6}{|c|}{ Is ET the same or different from the type of tremor that many normal people can get when they become old and frail? } & $p=0.020^{a}$ \\
\hline The same & $11(14.5)$ & $0(0.0)$ & $10(4.2)$ & 4 (10.3) & $25(5.9)$ & \\
\hline Similar but not the same & 20 (26.3) & $19(25.7)$ & $55(23.1)$ & $4(10.3)$ & $98(23.0)$ & \\
\hline Different & $34(44.7)$ & 36 (48.6) & 114 (47.9) & 20 (51.3) & $204(47.8)$ & \\
\hline Do not know & $11(14.5)$ & $19(25.7)$ & $58(24.4)$ & $11(28.2)$ & $99(23.2)$ & \\
\hline \multicolumn{6}{|l|}{ Is ET the same or different from Parkinson's disease? } & $p=0.127^{\mathrm{a}}$ \\
\hline The same & $1(1.3)$ & $1(1.4)$ & $0(0.0)$ & $0(0.0)$ & $2(0.5)$ & \\
\hline Different & $74(97.4)$ & $66(89.2)$ & $213(89.5)$ & $32(82.1)$ & $385(90.2)$ & \\
\hline Do not know & $1(1.3)$ & $7(9.5)$ & $24(10.1)$ & $7(17.9)$ & $39(9.1)$ & \\
\hline \multicolumn{6}{|l|}{ What is the typical age of onset of ET? } & $p=0.006^{a}$ \\
\hline $1-10$ & $4(5.3)$ & $1(1.4)$ & $2(0.8)$ & $0(0.0)$ & $7(1.6)$ & \\
\hline $11-20$ & $3(3.9)$ & $11(14.9)$ & $4(1.7)$ & $2(5.1)$ & $20(4.7)$ & \\
\hline $21-30$ & 7 (9.2) & $6(8.1)$ & $14(5.9)$ & $2(5.1)$ & $29(6.8)$ & \\
\hline $31-40$ & $3(3.9)$ & $4(5.4)$ & $15(6.3)$ & $1(2.6)$ & $23(5.4)$ & \\
\hline $41-50$ & $8(10.5)$ & $8(10.8)$ & 30 (12.6) & $3(7.7)$ & $49(11.5)$ & \\
\hline $51-60$ & 8 (10.5) & $6(8.1)$ & $36(15.1)$ & 7 (17.9) & 57 (13.3) & \\
\hline $61-70$ & $4(5.3)$ & $6(8.1)$ & $17(7.1)$ & 5 (12.8) & $32(7.5)$ & \\
\hline $71-80$ & $1(1.3)$ & $0(0.0)$ & $6(2.5)$ & $0(0.0)$ & 7 (1.6) & \\
\hline Any age & 25 (32.9) & $14(18.9)$ & $58(24.4)$ & 5 (12.8) & 102 (23.9) & \\
\hline Do not know & $11(14.5)$ & $17(23.0)$ & 49 (20.6) & $14(35.9)$ & 91 (21.3) & \\
\hline \multicolumn{6}{|l|}{ Can children get ET? } & $p<0.001^{a}$ \\
\hline Yes & 58 (76.3) & 47 (63.5) & 91 (38.2) & $10(25.6)$ & 206 (48.2) & \\
\hline No & $2(2.6)$ & $2(2.7)$ & $13(5.5)$ & $1(2.6)$ & $18(4.2)$ & \\
\hline Do not know & $16(21.1)$ & 25 (33.8) & 134 (56.3) & $28(71.8)$ & 203 (47.5) & \\
\hline \multicolumn{6}{|c|}{ On average, the tremor of ET progresses (worsens) at a rate of what percent per year? } & $p=0.927^{\mathrm{a}}$ \\
\hline It is not progressive & $13(17.1)$ & $23(31.1)$ & $60(25.2)$ & $9(23.1)$ & $105(24.6)$ & \\
\hline$<1 \%$ & $9(11.8)$ & $7(9.5)$ & $24(10.1)$ & $5(12.8)$ & $45(10.5)$ & \\
\hline $1-5 \%$ & $29(38.2)$ & $25(33.8)$ & $89(37.4)$ & $14(35.9)$ & $157(36.8)$ & \\
\hline $6-10 \%$ & $11(14.5)$ & $10(13.5)$ & $34(14.3)$ & $6(15.4)$ & $61(14.3)$ & \\
\hline $11-15 \%$ & $4(5.3)$ & $1(1.4)$ & $13(5.5)$ & $1(2.6)$ & $19(4.4)$ & \\
\hline $16-20 \%$ & $1(1.3)$ & $1(1.4)$ & $6(2.5)$ & $0(0.0)$ & $8(1.9)$ & \\
\hline $21-25 \%$ & $1(1.3)$ & $0(0.0)$ & $1(0.4)$ & $0(0.0)$ & $2(0.5)$ & \\
\hline More than 25\% & $3(3.9)$ & $3(4.1)$ & $5(2.1)$ & $1(2.6)$ & $12(2.8)$ & \\
\hline Don't know & $5(6.6)$ & $4(5.4)$ & $6(2.5)$ & $3(7.7)$ & $18(4.2)$ & \\
\hline \multicolumn{6}{|c|}{ Do patients with ET have an increased mortality compared to people without ET? } & $p=0.029^{a}$ \\
\hline No & $46(60.5)$ & $36(48.6)$ & $105(44.1)$ & $10(25.6)$ & $197(46.1)$ & \\
\hline Yes, but only slightly & $6(7.9)$ & $4(5.4)$ & $23(9.7)$ & $3(7.7)$ & $36(8.4)$ & \\
\hline Yes, and a lot & $1(1.3)$ & $0(0.0)$ & $1(0.4)$ & $1(2.6)$ & $3(0.7)$ & \\
\hline Don't know & $23(30.3)$ & $34(45.9)$ & $109(45.8)$ & $25(64.1)$ & $191(44.7)$ & \\
\hline \multicolumn{6}{|c|}{ Do you think people with ET die at a younger age than people who do not have ET? } & $p=0.215^{\mathrm{a}}$ \\
\hline Yes & $2(2.6)$ & $3(4.1)$ & $5(2.1)$ & $2(5.1)$ & $12(2.8)$ & \\
\hline No & $56(73.7)$ & $46(62.2)$ & $157(66.0)$ & $19(48.7)$ & $278(65.1)$ & \\
\hline Do not know & $18(23.7)$ & $25(33.8)$ & $76(31.9)$ & $18(46.2)$ & $137(32.1)$ & \\
\hline \multicolumn{6}{|c|}{ What do you think is the average memory/thinking deficit of a patient with ET? } & $p=0.086^{a}$ \\
\hline No problems with memory/thinking & $57(75.0)$ & $50(67.6)$ & $193(81.1)$ & $28(71.8)$ & $328(76.8)$ & \\
\hline Mild problem & $19(25.0)$ & $20(27.0)$ & $37(15.5)$ & $9(23.1)$ & 85 (19.9) & \\
\hline Moderate problem & $0(0.0)$ & $4(5.4)$ & $7(2.9)$ & $1(2.6)$ & $12(2.8)$ & \\
\hline Severe problem (dementia) & $0(0.0)$ & $0(0.0)$ & $1(0.4)$ & $1(2.6)$ & $2(0.5)$ & \\
\hline \multicolumn{6}{|l|}{ Is ET linked in any way with hearing loss? } & $p=0.022^{\mathrm{a}}$ \\
\hline Yes & $0(0.0)$ & $1(1.4)$ & $11(4.6)$ & $1(2.6)$ & $13(3.0)$ & \\
\hline No & $36(47.4)$ & $34(45.9)$ & $84(35.3)$ & 8 (20.5) & $162(37.9)$ & \\
\hline Don't know & $40(52.6)$ & $39(52.7)$ & $143(60.1)$ & 30 (76.9) & 252 (59.0) & \\
\hline \multicolumn{6}{|l|}{ Is ET linked in any way with sleeping problems? } & $p=0.030^{a}$ \\
\hline Yes & $11(14.5)$ & $8(10.8)$ & 46 (19.3) & 4 (10.3) & 69 (16.2) & \\
\hline No & 25 (32.9) & 18 (24.3) & $48(20.2)$ & 4 (10.3) & 95 (22.2) & \\
\hline Don't know & $40(52.6)$ & 48 (64.9) & $144(60.5)$ & 31 (79.5) & 263 (61.6) & \\
\hline
\end{tabular}




\begin{tabular}{|c|c|c|c|c|c|c|}
\hline & Proband & $\begin{array}{l}\text { Affected } \\
\text { relative }\end{array}$ & $\begin{array}{l}\text { Unaffected } \\
\text { relative }\end{array}$ & $\begin{array}{c}\text { Spouse of } \\
\text { unaffected relative }\end{array}$ & All participants & Significance \\
\hline \multicolumn{7}{|c|}{ Which of the following medical conditions do you think could be related in some way to ET? } \\
\hline Epilepsy & $8(10.5)$ & $7(9.5)$ & $34(14.3)$ & $5(12.8)$ & $54(12.6)$ & $p=0.666^{\mathrm{a}}$ \\
\hline Multiple sclerosis & $5(6.6)$ & $5(6.8)$ & $14(5.9)$ & $0(0.0)$ & $24(5.6)$ & $p=0.446^{\mathrm{a}}$ \\
\hline Parkinson's disease & $28(36.8)$ & $23(31.1)$ & $102(42.9)$ & $15(38.5)$ & 168 (39.3) & $p=0.313^{\mathrm{a}}$ \\
\hline Alzheimer's disease & $3(3.9)$ & $5(6.8)$ & $12(5.0)$ & $2(5.1)$ & $22(5.2)$ & $p=0.891^{\mathrm{a}}$ \\
\hline Neuropathy & $9(11.8)$ & $8(10.8)$ & $36(15.1)$ & $5(12.8)$ & 58 (13.6) & $p=0.757^{\mathrm{a}}$ \\
\hline Dystonia & $17(22.4)$ & $9(12.2)$ & $38(16.0)$ & $4(10.3)$ & 68 (15.9) & $p=0.253^{\mathrm{a}}$ \\
\hline More than one of the above & $0(0.0)$ & $3(4.1)$ & $0(0.0)$ & $0(0.0)$ & $3(0.7)$ & $p=0.002^{\mathrm{a}}$ \\
\hline Uncertain & $21(27.6)$ & $35(47.3)$ & $108(45.4)$ & $19(48.7)$ & $183(42.9)$ & $p=0.030^{a}$ \\
\hline Is Parkinson's disease linked in any way with ET? & & & & & & $p<0.001^{a}$ \\
\hline Yes & $13(17.1)$ & $6(8.1)$ & $41(17.2)$ & $5(12.8)$ & $65(15.2)$ & \\
\hline No & $42(55.3)$ & 25 (33.8) & $69(29.0)$ & $10(25.6)$ & $146(34.2)$ & \\
\hline Don't know & $21(27.6)$ & $43(58.1)$ & $128(53.8)$ & $24(61.5)$ & $216(50.6)$ & \\
\hline \multicolumn{6}{|c|}{ If a person has ET, what is their risk of developing Parkinson's disease? } & $p=0.370^{\mathrm{a}}$ \\
\hline The same as someone without ET & $61(80.3)$ & $63(85.1)$ & $199(83.6)$ & $31(79.5)$ & $354(82.9)$ & \\
\hline $1.5 \times$ higher than someone without ET & $8(10.5)$ & $2(2.7)$ & $11(4.6)$ & $5(12.8)$ & $26(6.1)$ & \\
\hline $2 \times$ higher than someone without ET & $2(2.6)$ & $5(6.8)$ & $9(3.8)$ & $1(2.6)$ & $17(4.0)$ & \\
\hline $3 \times$ higher than someone without ET & $0(0.0)$ & $1(1.4)$ & $3(1.3)$ & $0(0.0)$ & $4(0.9)$ & \\
\hline $4 \times$ higher than someone without ET & $1(1.3)$ & $0(0.0)$ & $3(1.3)$ & $0(0.0)$ & $4(0.9)$ & \\
\hline $5 \times$ higher than someone without ET & $0(0.0)$ & $0(0.0)$ & $1(0.4)$ & $1(2.6)$ & $2(0.5)$ & \\
\hline Less than someone without ET & $1(1.3)$ & $3(4.1)$ & $8(3.4)$ & $0(0.0)$ & $12(2.8)$ & \\
\hline Don't know & $2(2.6)$ & $0(0.0)$ & $1(0.4)$ & $1(2.6)$ & $4(0.9)$ & \\
\hline \multicolumn{7}{|l|}{ What types of doctors general take care of a patient with ET? } \\
\hline General doctor (general practitioner) & $27(35.5)$ & $14(18.9)$ & $72(30.3)$ & $6(15.4)$ & $119(27.9)$ & $p=0.031$ \\
\hline Internist & $9(11.8)$ & $2(2.7)$ & $19(8.0)$ & $3(7.7)$ & $33(7.7)$ & $p=0.217$ \\
\hline Neurologist & $72(94.7)$ & $65(87.8)$ & $205(86.1)$ & $34(87.2)$ & $376(88.1)$ & $p=0.252$ \\
\hline Neurosurgeon & $20(26.3)$ & $8(10.8)$ & $33(13.0)$ & $4(10.3)$ & 65 (15.2) & $p=0.024$ \\
\hline Other & $2(2.6)$ & $1(1.4)$ & $2(0.8)$ & $1(2.6)$ & $6(1.4)$ & $p=0.625$ \\
\hline Not sure & $2(2.6)$ & $9(12.2)$ & $34(14.3)$ & $5(12.8)$ & $50(11.7)$ & $p=0.054$ \\
\hline More than one of the above & $33(43.4)$ & $20(27.0)$ & $98(41.2)$ & $10(25.6)$ & $161(37.7)$ & $p=0.040$ \\
\hline \multicolumn{6}{|l|}{ Do you think the symptoms of ET can be medically controlled? } & $p=0.006^{a}$ \\
\hline Yes, very well & $4(5.3)$ & $6(8.1)$ & $3(1.3)$ & $0(0.0)$ & $13(3.0)$ & \\
\hline Yes, with moderate success & $35(46.1)$ & $25(33.8)$ & $87(36.6)$ & $13(33.3)$ & $160(37.5)$ & \\
\hline Yes, but not well & $19(25.0)$ & $10(13.5)$ & $67(28.2)$ & $8(20.5)$ & $104(24.4)$ & \\
\hline No & $8(10.5)$ & $10(13.5)$ & $22(9.2)$ & $3(7.7)$ & $43(10.1)$ & \\
\hline Do not know & $10(13.2)$ & $23(31.1)$ & $59(24.8)$ & $15(38.5)$ & $107(25.1)$ & \\
\hline Is there some type of brain surgery to treat ET? & & & & & & $p=0.001^{a}$ \\
\hline Yes & $55(72.4)$ & $38(51.4)$ & $115(48.3)$ & $14(35.9)$ & $222(52.0)$ & \\
\hline No & $5(6.6)$ & $9(12.2)$ & $17(7.1)$ & $3(7.7)$ & $34(8.0)$ & \\
\hline Do not know & $16(21.1)$ & $27(36.5)$ & $106(44.5)$ & $22(56.4)$ & $171(40.0)$ & \\
\hline Could diet and exercise prevent ET or help to control it? & & & & & & $p<0.001^{a}$ \\
\hline Yes & $18(23.7)$ & $16(21.6)$ & $33(13.9)$ & $11(28.2)$ & $78(18.3)$ & \\
\hline No & $29(38.2)$ & $22(29.7)$ & $47(19.7)$ & $5(12.8)$ & $103(24.1)$ & \\
\hline Do not know & $29(38.2)$ & $36(48.6)$ & $158(66.4)$ & $23(59.0)$ & $246(57.6)$ & \\
\hline Do you think ET is a curable disease? & & & & & & $p=0.002^{a}$ \\
\hline Yes & $6(7.9)$ & $4(5.4)$ & $14(5.9)$ & $5(12.8)$ & $29(6.8)$ & \\
\hline No & $51(67.1)$ & $34(45.9)$ & $124(52.1)$ & $10(25.6)$ & $219(51.3)$ & \\
\hline Do not know & $19(25.0)$ & $36(48.6)$ & $100(42.0)$ & $24(61.5)$ & $179(41.9)$ & \\
\hline Do you know of a celebrity or historical figure with ET? & & & & & & $p=0.001^{\mathrm{a}}$ \\
\hline Yes & $33(43.4)$ & $16(21.6)$ & $57(23.9)$ & $5(12.8)$ & $111(26.0)$ & \\
\hline No & $43(56.6)$ & $58(78.4)$ & $181(76.1)$ & $34(87.2)$ & $316(74.0)$ & \\
\hline
\end{tabular}

Values are numbers (percentages).

Significant $p$ values are in bold ( $p<0.05)$.

${ }^{a}$ Chi-square test or Fisher's exact test.

63.2\%) thought that ET patients have more problems with balance and walking than people without ET (Table 2).

\section{Distinction from Other Conditions}

Nearly one-third of probands answered "yes" or "do not know" to the question, "is ET the same or different from the type of tremor that many normal people can get when they become old and frail?" (Table 2). Although a full $90.2 \%$ of participants (385/427) reported that ET is different from $\mathrm{PD}, 10 / 150$ (6.7\%) participants with ET (i.e., probands and AFRs) thought the two diseases were the same or did not know. Interestingly, as many as 7/39 (17.9\%) spouses did not know (Table 2).

\section{Natural History and Prognosis}

Nearly one-half (206/427, 48.2\%) of participants acknowledged that children could get ET. Nearly one-third of probands (18/76, 
23.6\%) either did not know or responded "no," and three-fourth of spouses $(29 / 39,74.4 \%)$ did the same (Table 2$)$, with a trend across the four groups (test for trend for "do not know" $p<0.001$ ). Most probands responded that ET was progressive, and indicated that the rate of progression was gradual, with the rate most commonly endorsed being 1-5\% per year (Table 2). Approximately one-third [23/76 (30.3\%)] of probands did not know whether patients with ET have an increased risk of mortality (Table 2) and there was a clear trend across the four groups (30.3\% of probands, $45.9 \%$ of AFRs, $45.8 \%$ of UFRs, and $64.1 \%$ of spouses, test for trend $p=0.002$ ). The results were similar with the more direct question as to whether ET cases die at a younger age than those without ET (Table 2).

\section{Comorbidities}

Three-quarters [328/427 (76.8\%)] of our participants did not think that patients with ET had cognitive difficulty and virtually none $[2 / 427(0.5 \%)]$ thought that patients with ET experience dementia (Table 2). Only thirteen (3.0\%) participants thought that ET was linked with hearing loss and only $1 / 150(0.7 \%)$ affecteds (i.e., probands and AFRs) thought so (Table 2). Similarly, few positively endorsed the presence of sleep problems in ET; more than $60 \%$ of participants [263/427 $(61.6 \%)]$ were uncertain if ET has any links to sleep problems. When asked which medical conditions were related to ET in some way, ET was most commonly linked with PD $(36.8 \%$ of probands) and with dystonia (22.4\% of probands); these values were higher than those for multiple sclerosis, epilepsy, and neuropathy (Table 2). Despite this, the majority of participants $(354 / 427,82.9 \%)$ and probands $(61 / 76,80.3 \%)$ indicated that ET patients were not at increased risk of developing PD.

\section{Treatments}

Few $[4 / 76$ (5.3\%)] probands thought that the symptoms of ET could be very well controlled medically. Interestingly, 33/150 (22.0\%) of affecteds (i.e., probands and AFRs) did not know whether or to what degree (e.g., very well, moderately well, not well) the symptoms of ET could be medically controlled.

Of the affecteds, 57/150 (38.0\%) reported that there was no brain surgery for ET or that they did not know. For those who endorsed that there was brain surgery, there was a clear trend across groups (Table 2) (test for trend, $p<0.001$ ).

\section{Other}

The majority $(316 / 427,74.0 \%)$ of respondents did not know of a celebrity or historical figure with ET (Table 2) and there was a trend across the four groups (test for trend, $p<0.001$ ).

\section{Additional Analyses}

Sixty participants (9 probands, 6 AFRs, 41 UFRs, and 4 spouses) were in a health-related occupation. Because this may have biased the entire sample toward greater levels of knowledge, we repeated our main analyses in the subsample of 367 participants who were not in a health-related occupation. Answers to the questions about the body parts that shake, whether ET is different from PD, whether genes are a cause of ET, whether ET can be medically controlled, and whether there is brain surgery for ET were similar in this group (data not shown).

Affected relatives comprised both first- and second-degree relatives and we tested to see whether level of knowledge was higher in the former. Above we highlighted seven questions for which there was a trend across our four groups. For five of seven of these questions, the AFRs who were first-degree provided responses that exhibited greater knowledge than the AFRs who were second-degree relatives.

We also tested whether severity of tremor in the dominant arm affected knowledge, although did not subject the comparisons to statistical testing due to small sample size. For most comparisons, those with severe tremor had slightly greater knowledge than those with milder tremor. Thus, there were 17 probands who had a tremor rating of 3 on spiral drawing (i.e., severe tremor). Sixteen $(94.1 \%)$ of these endorsed genes as a cause of ET [compared to $51 / 59$ (86.4\%) probands with tremor rating $<3$ ]. Among 17 probands with severe tremor, 5 (29.4\%) did not know the level to which first-degree relatives of affected persons are at increased risk for ET; among the 59 probands with milder tremor, this value was $26(44.1 \%)$. Fifteen $(88.2 \%)$ of 17 probands with severe tremor endorsed that there was brain surgery for ET compared with $40(67.8 \%)$ of 59 probands with milder tremor. Three (17.6\%) of 17 probands with severe tremor vs. 20 (33.9\%) of probands with milder tremor did not know whether patients with ET have an increased risk of mortality. However, for several comparisons, those with more severe tremor did not seem to have more knowledge than those with milder tremor. For example, $15(88.2 \%)$ of 17 probands with severe tremor identified cranial tremor as a part of the body that could be involved vs. 54/59 (91.5\%) with milder tremor. Seven $(41.2 \%)$ of 17 probands with severe tremor vs. $11(18.4 \%)$ of 59 probands with milder tremor either did not know or responded "no" to the question about children getting ET. Seven (41.2\%) of 17 probands with severe tremor vs. 26 (44.1\%) of 59 with milder tremor knew of a celebrity or historical figure with ET.

\section{DISCUSSION}

Essential tremor is one of the most prevalent neurological diseases in the United States (1). However, it is still a poorly understood disease, especially among the public (11). As such, it does not stand alone. Epilepsy, another highly prevalent neurological disease, is also poorly understood by the public. A mail survey of 4,397 individuals in the United States indicated that the public, in general, had relatively little knowledge of epilepsy (13). Studies of family members of epilepsy patients tend to reveal relatively higher levels of knowledge. A survey of 124 first-degree relatives of epilepsy patients attending a tertiary clinic in Iran indicated that $50 \%$ had "good" knowledge about the disease, defined as having a high score on a 25 -item questionnaire (14).

Studies similar to ours, of ET patients and/or their families, are few. Fifty ET patients seen by neurologists at ColumbiaPresbyterian Medical Center completed a brief survey to assess their knowledge of the genetics of ET, revealing that the majority of these patients were not well informed (15). Similarly, a survey of 111 ET patients and their relatives at a tertiary care center in 
Singapore concluded that families had little knowledge about ET genetics (10). The authors attributed this result to the possibility that physicians might not be disseminating information because they might "consider ET as 'benign' disorder of less urgency or they may be ignorant about the etiology as well" (10). A survey of 250 patients attending various outpatient clinics at Yale New Haven Hospital, including neurology patients and PD patients, concluded that public knowledge about ET was poor (11). No studies have administered a comprehensive questionnaire about the features of ET to ET patients themselves and none has focused exclusively on those with familial ET, which is a group more likely to be knowledgeable about ET.

In the current study, we found that ET patients and their families knew the most basic, observable features of ET (e.g., the presence of hand tremors). However, as the questionnaire progressed through a range of questions about non-motor symptoms in ET, pathophysiology, and prognosis, family members became more uncertain. In other words, knowledge that is being generated in the scientific community is not necessarily filtering down to patients and their families.

We would expect questions regarding genetics and heredity to pique extra interest among family members. However, $16.7 \%$ of affecteds did not endorse genes as a cause for ET and this is surprising given the fact that this was a family study of ET whose stated goal in recruitment materials was to try to identify genes for ET. Virtually no participants were familiar with specific genes that have been reported as associated with ET in scientific studies (16-19). Forty percent of probands did not know the degree of increased risk in first-degree relatives of affected persons; published data have indicated that first-degree relatives of ET cases are five times more likely to develop the disease than are members of the general population and 10 times more likely if the proband's tremor began at an early age (20). While it is possible that patients and families are unaware of current scientific data, the results of gene finding efforts in ET have been mixed (21) and it is possible that ET patients are aware of scientific studies but unable to understand or recall the results.

The results suggest that ET patients lack knowledge even about its clinical features, its natural history, and its distinction from other conditions. Nearly 1 in 3 probands either thought ET was the same as the type of tremor that many normal people can get when they become old and frail or did not know. That is, they did not distinguish the disease from an age-associated trait of normal aging. Nearly 1 in 3 probands either did not know whether children could get ET or responded "no." Nearly 1 in 4 affecteds (i.e., probands and AFRs) did not know whether ET could be medically controlled and $38.0 \%$ of affecteds reported that there was no brain surgery for ET or that they did not know. This may be indicative of poor patient education about the disease and treatment options. Previous studies mentioned this deficiency and the patient's need for more knowledgeable physicians. For example, a poll of 1,418 ET patients revealed that $31.4 \%$ of respondents felt that their doctor was not even moderately well-educated about ET (6).

Probands and AFRs were groups that were most aware of the basic features of ET and were least likely to answer "I don't know" to questions, suggesting they are more confident in their answers. They may be more motivated to learn more about their disease.
When compared with other groups, spouses of unaffected relatives appeared to be the most uninformed and were more likely to answer "I don't know" on most questions.

Compared to the data presented in Shalaby's study (11), spouses in this study seem to have more general ET knowledge than the public. This is not unexpected, as they have a family member with ET, although not necessarily a close one. This suggests that some knowledge of the condition is circulating through families and is even reaching spouses of the unaffected relatives. For example, on the question, "Is ET the same or different from PD?" two-third of Shalaby's participants [69/99 (69.7\%)] noted that ET is different from PD while in this study four-fifth of spouses [32/39 (82.1\%)] believed the two diseases were different. When asked, "what parts of the body can shake when someone has ET?" 38 (97.4\%) spouses identified arms and 23 (59.0\%) identified cranial structures. Among Shalaby's participants, 66 (66.7\%) identified arms and 42 (42.4\%) identified cranial structures. However, on more advanced topics, such as etiology, the spouses' knowledge appears similar to the general public's (11). One caveat, however, with regard to the comparison of the present data to those from Shalaby's study (11) is that different populations were studied (i.e., the current data were from a family study whereas those of Shalaby were from a survey of 250 patients attending various outpatient clinics at Yale New Haven Hospital). Also, the questionnaires used in the two studies were not identical. These factors could also have contributed to differences between the two studies.

We also tested whether severity of tremor in the dominant arm affected level of knowledge. For most comparisons, those with severe tremor had slightly greater knowledge than those with milder tremor. This is understandable; individuals with more tremor-related dysfunction are more likely as a group to be motivated to learn about their condition.

This study draws attention to a gap in knowledge about ET. One must ask why this gap exists. First, the extent to which this gap in knowledge is derived from the level of knowledge of physicians regarding ET is not clear. How much does the general physician know about ET? How much does the average neurologist know about ET? How much does the typical movement disorders neurologist know about ET? To adequately educate patients and families, physicians must be well aware of recent advances in ET. As a corollary, physicians may be less prone to educate patients regarding issues that are not well established or are controversial, including the diagnosis of ET, its separation from age-related conditions and its association with other degenerative conditions. Second, ET may not be very disabling for some patients, and these patients may be less willing to gain knowledge about the disease. Third, public awareness and social media coverage of ET is less than that of PD. Fourth, the neurological literature is dominated by PD compared to ET.

Are there methods one can use to improve knowledge of ET? The first step is drawing attention to the problem. The second is devising strategies to deal with it. These may include public awareness campaigns (e.g., ET awareness month) as well as awareness campaigns to educate physicians (e.g., distribution of literature, sponsoring of seminars). Although patient advocacy groups are actively engaged in these efforts, lack of significant 
financial support on the part of the ET community has limited the scope of these efforts. Another problem in ET is the lack of a public figure who is affected and is willing to serve as a public spokesperson for the disease.

The current study should be interpreted within the context of certain limitations. Although the strength of the study was its family design, familial ET cases are not representative of all ET cases, as their knowledge of ET is expected to be higher. Furthermore, collection of data from the public as well as from neurologists would provide additional insights about more widespread knowledge of ET. Also, although the sample size was large, the numbers in certain groups (e.g., spouses) was smaller. Finally, some of the questions and the topics of the questionnaire were difficult and complex (e.g., the names of specific genes) and this could have impacted the ability of patients to demonstrate their knowledge.

Strengths of the study include the overall sample size $(n=427)$. Second, is the uniqueness of the questionnaire and the fact that this is the first study to administer a comprehensive questionnaire to ET patients themselves. Third, it is the first study to focus exclusively on those with familial ET, to gauge the level of knowledge in a group with expected high levels of knowledge. Finally, we surveyed four different types of individuals who a priori were expected to exhibit a graded range of knowledge of ET, from probands, who were expected to have the most, to spouses of unaffected relatives, who were expected to have the least.

In conclusion, we targeted a large group of ET patients and their families, as this group is perhaps most likely to be knowledgeable about the disease. ET patients and their AFRs were more knowledgeable about the features of ET compared to their family members who do not have ET. Overall, however, knowledge of ET was very limited and this lack of knowledge encompassed all aspects of the disease, including its underlying causes, the nature of the symptoms and signs, its natural history, and its treatment. Further ET awareness education and programs targeting both families of ET patients and the public would help alleviate this gap in knowledge.

\section{ETHICS STATEMENT}

This study was carried out in accordance with the recommendations of the Yale University Institutional Review Board with written informed consent from all subjects. All subjects gave written informed consent in accordance with the Declaration of Helsinki.

\section{REFERENCES}

1. Louis ED, Ferreira JJ. How common is the most common adult movement disorder? Update on the worldwide prevalence of essential tremor. Mov Disord (2010) 25(5):534-41. doi:10.1002/mds.22838

2. Louis ED. Essential tremor then and now: how views of the most common tremor diathesis have changed over time. Parkinsonism Relat Disord (2017) 46 Suppl 1:S70-4. doi:10.1016/j.parkreldis.2017.07.010

3. Benito-León J. Essential tremor: from a monosymptomatic disorder to a more complex entity. Neuroepidemiology (2008) 31(3):191-2. doi:10.1159/ 000154933

4. Jain S, Lo SE, Louis ED. Common misdiagnosis of a common neurological disorder: how are we misdiagnosing essential tremor? Arch Neurol (2006) 63(8):1100-4. doi:10.1001/archneur.63.8.1100
The protocol was approved by the Yale University Institutional Review Board.

\section{AUTHOR CONTRIBUTIONS}

AC was involved in the conception and design of this work, the statistical analysis and interpretation of data, the drafting of the manuscript, and gives final approval of this version to be published and agreement to be accountable for all aspects of the work in question. $\mathrm{KC}$ was involved in the acquisition of data, the critical revision of the manuscript, and gives final approval of the version to be published and agreement to be accountable for all aspects of the work in question. $\mathrm{NH}$ was involved in the acquisition of data, the critical revision of the manuscript, and gives final approval of the version to be published and agreement to be accountable for all aspects of the work in question. PF-L was involved in the conception and design of this work, the critical revision of the manuscript, and gives final approval of the version to be published and agreement to be accountable for all aspects of the work in question. LC was involved in the conception and design of this work, the critical revision of the manuscript, and gives final approval of the version to be published and agreement to be accountable for all aspects of the work in question. RO was involved in the conception and design of this work, the acquisition of data, the critical revision of the manuscript, and gives final approval of the version to be published and agreement to be accountable for all aspects of the work in question. EL was involved in the conception and design of this work, the analysis and interpretation of data, the drafting of the manuscript, and gives final approval of this version to be published and agreement to be accountable for all aspects of the work in question.

\section{FUNDING}

EL has received research support from the National Institutes of Health: Grant numbers NINDS R01 NS094607 and NINDS R01 NS073872 (principal investigator), NINDS \#R01 NS085136 (principal investigator), NINDS \#R01 NS073872 (principal investigator), NINDS \#R01 NS085136 (principal investigator), and NINDS \#R01 NS088257 (principal investigator). He has also received support from the Claire O'Neil Essential Tremor Research Fund (Yale University). This funding body played no role in the design of the study, the collection, analysis, and interpretation of data, or the writing of the manuscript.

5. Schrag A, Munchau A, Bhatia KP, Quinn NP, Marsden CD. Essential tremor: an overdiagnosed condition? J Neurol (2000) 247(12):955-9. doi:10.1007/ s004150070053

6. Louis ED, Rohl B, Rice C. Defining the treatment gap: what essential tremor patients want that they are not getting. Tremor Other Hyperkinet Mov (N Y) (2015) 5:331. doi:10.7916/D87080M9

7. Jorgensen CR, Thomsen TG, Ross L, Dietz SM, Therkildsen S, Groenvold $\mathrm{M}$, et al. What facilitates "patient empowerment" in cancer patients during follow-up: a qualitative systematic review of the literature. Qual Health Res (2018) 28(2):292-304. doi:10.1177/1049732317721477

8. Schulz PJ, Nakamoto K. Health literacy and patient empowerment in health communication: the importance of separating conjoined twins. Patient Educ Couns (2013) 90(1):4-11. doi:10.1016/j.pec.2012. 09.006 
9. Louis ED, Machado DG. Tremor-related quality of life: a comparison of essential tremor vs. Parkinson's disease patients. Parkinsonism Relat Disord (2015) 21(7):729-35. doi:10.1016/j.parkreldis.2015.04.019

10. Prakash KM, Lum SY, Fook-Choong S, Tan EK. Knowledge about the genetics of essential tremor in patients and their relatives. Eur J Neurol (2006) 13(11):1266-9. doi:10.1111/j.1468-1331.2006.01517.x

11. Shalaby S, Indes J, Keung B, Gottschalk CH, Machado D, Patel A, et al. Public knowledge and attitude toward essential tremor: a questionnaire survey. Front Neurol (2016) 7:60. doi:10.3389/fneur.2016.00060

12. Louis ED, Badejo FM, Cristal AD, Meyers J, Hernandez N, Chen KP, et al. Early head tremor in essential tremor: a case series and commentary. Tremor Other Hyperkinet Mov (N Y) (2017) 7:453. doi:10.7916/D8KW5MRG

13. Kobau R, Price P. Knowledge of epilepsy and familiarity with this disorder in the U.S. population: results from the 2002 HealthStyles Survey. Epilepsia (2003) 44(11):1449-54. doi:10.1046/j.1528-1157.2003.17603.x

14. Karimi N, Akbarian SA. Knowledge and attitude toward epilepsy of close family members of people with epilepsy in north of Iran. Adv Med (2016) 2016:6. doi:10.1155/2016/8672853

15. Watner D, Jurewicz EC, Louis ED. Survey of essential tremor patients on their knowledge about the genetics of the disease. Mov Disord (2002) 17(2):378-81. doi:10.1002/mds.10085

16. Kuhlenbaeumer G, Tan EK, Wu YR. Identification of a novel risk variant in the FUS gene in essential tremor. Neurology (2014) 82(5):461. doi:10.1212/ WNL.0000000000000073

17. Liu X, Hernandez N, Kisselev S, Floratos A, Sawle A, Ionita-Laza I, et al. Identification of candidate genes for familial early-onset essential tremor. Eur J Hum Genet (2016) 24(7):1009-15. doi:10.1038/ejhg.2015.228
18. Merner ND, Girard SL, Catoire H, Bourassa CV, Belzil VV, Riviere JB, et al. Exome sequencing identifies FUS mutations as a cause of essential tremor. Am JHum Genet (2012) 91(2):313-9. doi:10.1016/j.ajhg.2012 07.002

19. Stefansson H, Steinberg S, Petursson H, Gustafsson O, Gudjonsdottir $\mathrm{IH}$, Jonsdottir GA, et al. Variant in the sequence of the LINGO1 gene confers risk of essential tremor. Nat Genet (2009) 41(3):277-9. doi:10.1038/ ng.299

20. Louis ED, Ford B, Frucht S, Barnes LF, X-Tang M, Ottman R. Risk of tremor and impairment from tremor in relatives of patients with essential tremor: a community-based family study. Ann Neurol (2001) 49(6):761-9. doi:10.1002/ ana. 1022

21. Testa CM. Key issues in essential tremor genetics research: where are we now and how can we move forward? Tremor Other Hyperkinet Mov (N Y) (2013) 3. doi:10.7916/D8Q23Z0Z

Conflict of Interest Statement: The authors declare that the research was conducted in the absence of any commercial or financial relationships that could be construed as a potential conflict of interest.

Copyright (c) 2018 Cristal, Chen, Hernandez, Factor-Litvak, Clark, Ottman and Louis. This is an open-access article distributed under the terms of the Creative Commons Attribution License (CC BY). The use, distribution or reproduction in other forums is permitted, provided the original author(s) and the copyright owner are credited and that the original publication in this journal is cited, in accordance with accepted academic practice. No use, distribution or reproduction is permitted which does not comply with these terms. 